\title{
HEAVY FLAVOR RESONANCES AND QED RADIATIVE CORRECTIONS*
}

\author{
James P. ALEXANDER, Giovanni BONVICINI, Persis S. DRELL, Ray FREY and Vera LÜTH \\ Lawrence Berkeley Laboratory, Berkeley CA 94720, USA \\ University of Michigan, Ann Arbor, MI 48109, USA \\ Stanford Linear Accelerator Center, Stanford University, Stanford CA 94309, USA
}

Received 15 February 1988

(Revised 23 January 1989)

\begin{abstract}
We discuss QED radiative corrections applied to narrow resonances in $\mathrm{e}^{+} \mathrm{e}^{-}$annihilation. We establish a simple and precise prescription for extracting radiative corrections from experimental data. This prescription differs from those used in measurements of charm and bottom resonances and leads to resonance parameters which are significantly different. Using a simulation method, we calculate these differences, and conclude that the masses and widths of $\psi$ and $\Upsilon$ resonances change by up to three standard deviations from presently accepted values.
\end{abstract}

\section{Introduction}

In this paper we examine the radiative corrections to very narrow resonances such as the $\psi$ and $\Upsilon$ particles. We focus on the determination of their mass $M$, their total width $\Gamma$, and their partial width to electrons $\Gamma_{\mathrm{c}}^{0}$. Our objective is to review experimental results that were obtained in analyses with incorrect radiative corrections. Our analysis shows in fact that the errors incurred are up to three times bigger than the uncertainties quoted for the current world averages [1].

In sect 1 , we present the prescription for radiative corrections used in our analysis. We briefly discuss why this treatment is a significant improvement over that used in most of the previous analyses of $\psi$ and $\Upsilon$ resonance data. In sect. 2 , we discuss in detail our method for correcting existing data, the effects of different algorithms on the extraction of the resonance parameters, and the effects of other possibly relevant differences between experiments. In sect. 3 , we use our prescription to refit the $\psi$ and $\Upsilon$ resonances, and extract new values for the resonance parameters in a way that is independent of the experimental conditions. Sect. 4 is

\footnotetext{
* Work supported by the Department of Energy, contracts DE-AC03-76SF00515 (SLAC), DE-AC0376SF00098 (LBL), and DE-AC02-76ER01112 (University of Michigan).
} 
dedicated to a discussion of the results, comparison with other results, and conclusions.

\section{Initial state radiative corrections to narrow resonances}

In $\mathrm{e}^{+} \mathrm{e}^{-}$collisions, the nominal collision energy, $\sqrt{s}=2 E$, is set by $E$, the energy of the incident beams. The actual c.m. energy available for the annihilation is reduced by bremsstrahlung to $\sqrt{s(1-k)}$, where $k E$ is the total energy of the emitted photons. The observed cross section, $\sigma_{\text {obs }}(s)$ at the nominal energy $\sqrt{s}$, can be written as a convolution of the Born cross section $\sigma_{0}(s(1-k))$ and a dimensionless sampling function $f(k, s)[2]$,

$$
\sigma_{\text {obs }}(s)=\int f(k, s) \sigma_{0}(s(1-k)) \mathrm{d} k
$$

In the vicinity of a quarkonium resonance we have

$$
\sigma_{0}=\sigma_{\text {nonres }}+\sigma_{\text {peak }} \frac{s \Gamma^{2}}{\left(s-M^{2}\right)^{2}+s \Gamma^{2}}
$$

where $M$ is the mass and $\Gamma$ is the total width of the resonance. It is well known that $f(k, s)$ is dominated by initial state effects [3]. Effects of final state radiation on the cross section are usually ignored at the fraction of a percent level.

We employ the following expression for $f(k, s)$, based on the results of Kuraev and Fadin [4], truncated to first order in the hard photon terms, and to second order in the vertex terms:

$$
f(k, s)=\left(1+\delta_{\mathrm{vp}}\right)(1+K)\left[\beta k^{\beta-1}\left(1+\delta_{1}+\delta_{2}\right)-\beta(1-k / 2)\right]
$$

where $\beta$ is the electron equivalent radiator thickness,

$$
\beta=\frac{2 \alpha}{\pi}\left(\log \frac{s}{m_{\mathrm{e}}^{2}}-1\right)
$$

The $\delta_{n}$ terms arise from the leading parts of the vertex correction diagrams of order $n, K$ is the $K$-factor, and $\delta_{\text {vp }}$ is due to the photon vacuum polarization. These terms are all reproduced in the appendix. We note that for the hadronic contribution to the vacuum polarization, $\delta_{h}$, we use values based on a calculation by Berends and Komen [5], namely $\delta_{\mathrm{h}}=1.1 \pm 0.5 \%$ at $\sqrt{s} \approx 3 \mathrm{GeV}$, and $3.4 \pm 1.0 \%$ at $\sqrt{s} \approx 10 \mathrm{GeV}$. The quoted uncertainties are our estimates. The uncertainty in $\delta_{\mathrm{h}}$ turns out to dominate the error in our calculations. 
In the past, most experimenters have fit the narrow resonances of the $\psi$ and $\Upsilon$ families using a different expression for $f(k, s)$, based on the classic work of Jackson and Scharre [6],

$$
f^{\prime}(k, s)=\delta_{\mathrm{tot}} \delta(k)+\beta k^{\beta-1}-\beta(1-k / 2), \quad \delta_{\mathrm{tot}}=\delta_{1}+\delta_{v p}^{\prime}+K .
$$

Here $\delta(k)$ is the Dirac function. This expression was obtained from a first order perturbative calculation with the inclusion of exponentiation of soft photons.

There are essential differences between the distribution functions $f(k, s)$ and $f^{\prime}(k, s)$. The differences occur in second order in $\alpha$. First, in the formulation by Jackson and Scharre, the photon vacuum polarization $\delta_{\mathrm{vp}}^{\prime}$ is approximated by the electron loop $\delta_{\mathrm{e}}$ only, excluding contributions from hadrons, muons, and $\tau$ leptons, $\delta_{\mathrm{h}}, \delta_{\mu}$, and $\delta_{\tau}$. Secondly, the vertex correction $\left(1+\delta_{1}\right)$ should multiply the bremsstrahlung term $k^{\beta-1}$, at least to first order, and hence should enter as an overall multiplicative factor to this term, as in eq. (3). Thirdly, the vacuum polarization should also enter as an overall multiplicative constant. The factorization of the virtual terms arises naturally from those semi-classical formalisms which are based on factorization principles [7,8]. This factorization of the virtual corrections can be checked to first order by doing an explicit second order calculation. A second order calculation [9] does not, however, determine unambiguously that the $\delta_{2}$ term factorizes, though it is a natural choice and it agrees with the Bloch-Nordsieck theorem [10]. In the definition of $f^{\prime}(k, s)$, the virtual corrections were not properly separated and the $\delta(k)$ terms gives a finite probability for the electron and positron to annihilate without soft photon emission, in direct disagreement with the Bloch-Nordsieck theorem. This locally distorts the cross section by a fraction $\approx 10 \%$ at $\sqrt{s}=3 \mathrm{GeV}$, and $\approx 14 \%$ at $\sqrt{s}=10 \mathrm{GeV}$.

The convolution integral of a Breit-Wigner resonance cross section with $f(k, s)$ can be solved analytically. We use the expression given in the appendix, which was derived by Cahn [11] for the $Z^{0}$ resonance. We have added the photon vacuum polarization and $\boldsymbol{\delta}_{2}$ terms. Since the energy spread of the incident beams, $\sigma_{E}$, is much greater than the resonance width, $\Gamma$, it dominates the shape of the visible resonance. We account for the energy spread by further convoluting the cross section with a gaussian resolution function of width $\sigma_{\mathrm{E}}$. The convolution is done by numerical integration. We believe that the error associated with our use of eq. (3) is about $1 \%$, coming mostly in normalization uncertainties associated with vacuum polarization.

\section{Distortion of the resonance shape and analysis method}

A resonance is described by its mass, $M$, and two of the following three parameters: the total width, $\Gamma$, the cross section integral $A$, and the cross section at 
the peak, $\sigma_{\text {peak }}$. These three parameters are related by the equation

$$
A=\frac{1}{2} \pi \Gamma \sigma_{\text {peak }}
$$

The observed width and maximum cross section are strongly influenced by the energy resolution $\sigma_{\mathrm{E}}$ and differ from the resonance parameters $\Gamma$ and $\sigma_{\text {peak }}$ by orders of magnitude.

In the following, we consider specifically the resonance cross section for the channel $\mathrm{e}^{+} \mathrm{e}^{-} \rightarrow$ hadrons. The area under the resonance, $A$, is related to the measured partial width to electrons, $\Gamma_{\mathrm{e}}^{\exp }$, and the branching ratio for this process, in our case $B_{\text {had }}$, by

$$
A=\frac{1}{2} \pi \Gamma \sigma_{\text {peak }}=\frac{6 \pi^{2}}{M^{2}} \Gamma_{\mathrm{e}}^{\mathrm{exp}} B_{\text {had }}, \quad \text { with } \quad B_{\text {had }}=\Gamma_{\text {had }} / \Gamma .
$$

Under the assumption, which has been experimentally verified [1], that the total width is the sum of the partial widths to hadrons and charged lepton pairs, and that the leptonic widths are all equal, we have

$$
\Gamma=\Gamma_{\mathrm{had}}^{\mathrm{exp}}+m \Gamma_{\mathrm{e}}^{\mathrm{exp}}, \text { and } m B_{\mathrm{e}}+B_{\mathrm{had}}=1 .
$$

Here $m$ stands for the number of partial widths into lepton pairs, $m=2$ for charmonium and $m=3$ for bottomonium states. The leptonic branching ratios are determined experimentally, and therefore the relations above can be used to measure the quantities $\Gamma$ and $\Gamma_{\mathrm{e}}^{\text {exp }}$.

We note explicitly the nature of $\Gamma_{\mathrm{e}}^{\mathrm{exp}}$, defined in eq. (8), and draw the distinction with the quantity of theoretical interest $[12], \Gamma_{\mathrm{c}}^{0}$. The physical coupling of the resonance to leptons through one photon is $\Gamma_{\mathrm{e}}^{\mathrm{exp}}$, which is obtained from the data by making all radiative corrections except vacuum polarization corrections. This is the quantity which, divided by the measured branching ratio, gives the total width. The value of $\Gamma_{\mathrm{e}}^{0}$, on the other hand, is drawn from the data by making all radiative corrections including vacuum polarization. Thus $\Gamma_{\mathrm{c}}^{0}$ reflects the coupling strength at tree level only. The quantity $\Gamma_{\text {had }}$, which couples to the resonance mostly through three gluons, does not have QED vacuum polarization corrections, and in this case $\Gamma_{\text {had }}^{\text {exp }}=\Gamma_{\text {had }}^{0}$.

Historically, experimenters have generally included some level of vacuum polarization in their corrections, and have therefore implicitly extracted $\Gamma_{\mathrm{c}}^{0}$. For the remainder of our discussion we follow this precedent, though at the end we include values for $\Gamma_{\mathrm{e}}^{\exp }$ in summary tables. The relationship between the two quantities is

$$
\Gamma_{\mathrm{e}}^{\exp }=\left(1+\delta_{\mathrm{vp}}\right) \Gamma_{\mathrm{e}}^{0}
$$




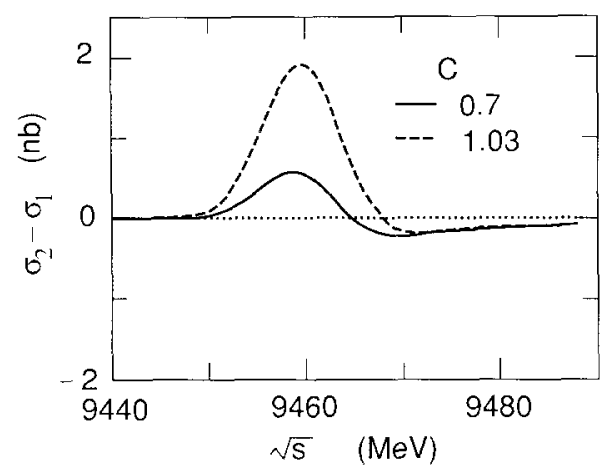

Fig. 1. The difference between the cross section for the $\Gamma(9460)$ calculated with $f(k, s)$ from eq. (3) and with $f^{\prime}(k, s)$ from eq. (5) using the same input parameters. The solid line represents the difference for the full vacuum polarization terms in $f^{\prime}(k, s)$, while the broken line gives the difference for only the electron contribution the vacuum polarization. Ratio $C$ is defined later in the text.

Since radiative effects in the final states are negligible, the branching ratios do not depend on radiative corrections. Thus differences in the formulation of the radiative corrections will cause changes in two parameters, the integral $A$ and the partial width $\Gamma_{\mathrm{e}}^{0}$. They will scale proportionally, with a factor that depends on the branching ratio for the particular channel under study. If one studies simultaneously the resonance cross sections into hadrons, muon pairs, and electron pairs, the three integrals will change by the same fraction, giving approximately the same change to $\Gamma_{\mathrm{e}}^{0}$, while the ratio between the three integrals (which determines the branching ratios) remains unchanged. Differences in the formulation of the functions $f(k, s)$ and $f^{\prime}(k, s)$ affect the resonance mass very little, at the level of one part in $10^{5}$.

The difference between our treatment of the radiative corrections and the formulation by Jackson and Scharre is illustrated in fig. 1. We plot the difference between the cross section for the $\Upsilon(9460)$ calculated with $f(k, s)$ and $f^{\prime}(k, s)$ using the same input parameters. The cross section is overestimated by $f^{\prime}(k, s)$ on and below the resonance, and is underestimated above the resonance. We illustrate both the case where the vacuum polarization in $f^{\prime}(k, s)$ includes all terms, $\delta_{\mathrm{vp}}^{\prime}=\delta_{\mathrm{e}}+\delta_{\mu}+\delta_{\tau}+\delta_{\mathrm{h}}$, and where it is reduced to the electron loop, $\delta_{\mathrm{vp}}^{\prime}=\delta_{\mathrm{e}}$. This latter case is the formulation that most previous experiments had used to fit narrow resonances. The use of the electron loop alone in the vacuum polarization reduces the difference in the predicted cross section at the peak resulting from the incorrect treatment of the virtual terms in $f^{\prime}(k, s)$.

Because the discrepancy between the two radiative correction schemes varies across the resonance, one must take into consideration the distribution of data points. Experiments collect most of their luminosity on the peak. When the data are fit, the $\chi^{2}$ weights the points on the peak strongly, so that shifts in the free parameters arising from the differences between $f(k, s)$ and $f^{\prime}(k, s)$ will be 
influenced most strongly by the discrepancy between the two functions in the vicinity of the resonance peak. This is further enhanced by fluctuations in the non-resonant cross section that can obscure the structure of the radiative tail of the resonance. Since $f^{\prime}(k, s)$ consistently overestimates the resonance area, fits based on this function compensate by underestimating $\Gamma_{\mathrm{e}}^{0}$. In addition, $f^{\prime}(k, s)$ suppresses the radiative tail and thereby leads to an overestimate of the non-resonant cross section, which is also a free parameter of the fit. The magnitudes of the shifts in the parameters obtained by the fit to the resonance will depend on details that will vary from experiment to experiment, such as the ratio of resonant to non-resonant cross section ( $\mathrm{R} / \mathrm{NR}$ ), the amount of integrated luminosity taken on the peak, and the energy spread of the machine.

At the $\Upsilon(9460)$, for example, we have $R / N R \approx 5$ and the radiative tail of the resonance is almost undetectable. The fitted values of $\Gamma_{\mathrm{e}}^{0}$ consequently reflect the large discrepancy in predictions of the peak height by the functions $f(k, s)$ and $f^{\prime}(k, s)$. Quantitatively, the fractional differences in the fitted values of $\Gamma_{\mathrm{e}}^{0}, \Delta \Gamma_{\mathrm{e}}^{0} / \Gamma_{\mathrm{e}}^{0}$, are almost equal to the fractional differences in the peak cross sections predicted by $f(k, s)$ and $f^{\prime}(k, s)$. Any increase in the machine energy spread, $\sigma_{\mathrm{E}}$, further enhances this effect as it further obscures the radiative tail. At the $J / \psi(3097)$, where the machine energy spread is smaller, the radiative enhancement to the tail of the resonance is clearly observable, and the fit is more sensitive to the underestimate of the cross section at the resonance tail by $f^{\prime}(k, s)$. The total correction to the resonance integral or $\Gamma_{\mathrm{e}}^{0}$ is significantly smaller than the difference in the peak heights.

The fractional difference in the total cross section (resonant plus non-resonant) for the $\Upsilon(9460)$ calculated using $f(k, s)$ and $f^{\prime}(k, s)$ with the same input parameters is shown in fig. 2. As in Fig. $1 f^{\prime}(k, s)$ is calculated both for $\delta_{\mathrm{vp}}^{\prime}=\delta_{\mathrm{e}}+\delta_{\mu}+\delta_{\tau}+$ $\delta_{\mathrm{h}}$ and $\delta_{\mathrm{vp}}^{\prime}=\delta_{\mathrm{e}}$. Limits on the total change in $\Gamma_{\mathrm{e}}^{0}$ from fitting with $f(k, s)$ and $f^{\prime}(k, s)$ can be derived directly from fig. 2 . The largest shift in $\Gamma_{\mathrm{e}}^{0}$ occurs if data points are taken only where the discrepancy is maximal (on the peak) and where it is minimal (about $20 \mathrm{MeV}$ above). In this somewhat contrived scenario, the data above the resonance fix the non-resonant cross section, and the points on resonance determine $\Gamma_{\mathrm{e}}^{0}$. Fig. 2 illustrates that the shift in $\Gamma_{\mathrm{e}}^{0}$ caused by using $f^{\prime}(k, s)$ instead of $f(k, s)$ to fit the data is $12 \%$ or $6 \%$, depending on whether the full or the reduced vacuum polarization is included in $f^{\prime}(k, s)$. In real experiments where the data points are more evenly distributed in energy, the changes in $\Gamma_{\mathrm{e}}^{0}$ will always be less than quoted above.

To correctly reproduce the complicated interplay of the fit parameters and to study the dependence and correlations among them, we resort to a technique of simulating the data obtained by various experiments to measure the $\psi$ and $\Upsilon$ resonances. We generate data points by calculating the cross section at a given energy $\sqrt{s}$ using our definition $f(k, s)$ and errors proportional to $\sqrt{\sigma_{\text {obs }}}$. Subsequently, the generated data points are fit by functions based on both $f(k, s)$ and 


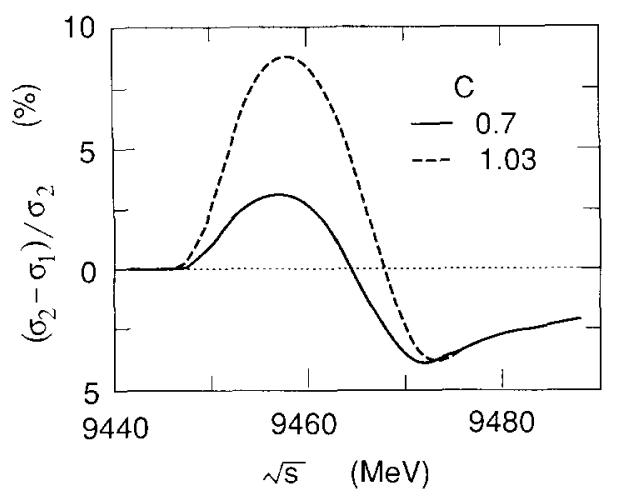

Fig. 2. A comparison of the calculated hadronic cross section near the $\Upsilon(9460)$ resonance. The curves represent the fractional difference between the calculations using $f(k, s)$ in eq. (3) and $f^{\prime}(k, s)$ in eq. (5) with the same input parameters. The solid line represents the difference for the full vacuum polarization terms in $f^{\prime}(k, s)$, while the broken line gives the difference for only the electron contribution the vacuum polarization. Ratio $C$ is defined later in the text.

$f^{\prime}(k, s)$. We study the changes to the fitted resonance parameters using the hadronic cross sections only.

For a compact presentation of the results in the following section we find it convenient to introduce the ratio

$$
C=\frac{\delta_{\mathrm{tot}}}{\delta_{1}+\delta_{\mathrm{vp}}+\delta_{2}+K},
$$

with $\delta_{\text {tot }}$ as defined in eq. (5). Hence, $C$ takes into account the inconsistent treatment of the vacuum polarization terms in the literature, as described above. The denominator is the correction to the soft bremsstrahlung term (i.e. the first term) in $f(k, s)$ when we assume the virtual terms are small. In the denominator, we take $\delta_{\mathrm{vp}}=\delta_{\mathrm{c}}+\delta_{\mu}+\delta_{\tau}+\delta_{\mathrm{h}}$, while the value of $\delta_{\mathrm{vp}}^{\prime}$ implicitly contained in $\delta_{\text {tot }}$ may be reduced to $\delta_{\mathrm{e}}$ as in the Jackson and Scharre ansatz. Using the ansatz we obtain $C=0.85$ and $C=0.70$ at $3.1 \mathrm{GeV}$ and $10 \mathrm{GeV}$, respectively. Using full vacuum polarization in $\delta_{\text {tot }}$ we obtain $C=1.03$. Since $\delta_{2}$ is not included in $\delta_{\text {tot }}$, the ratio $C$ is never quite equal to one.

In addition to our analysis of simulated resonance cross sections, we have also refit original Mark I data [13] on the $\psi(3097)$ and $\psi(3685)$ resonances to determine which of the two calculations of the radiative effects fit the data best. We analyze hadronic cross sections only and fix the leptonic branching ratio. For the $\psi(3097)$ we obtain $\chi^{2}$ per degree of freedom of $27.3 / 22$ and $30.9 / 22$ using $f(k, s)$ and $f^{\prime}(k, s)$, respectively. For the $\psi(3685)$ the corresponding results are $10.4 / 10$ and $12.4 / 10$. The two fitting functions yield different values for the parameters, but 
nearly the same values of $\chi^{2}$. Thus, the similar quality of the fits ensures that the extracted errors on the parameters are correct for both fits. Based on the existing data alone a discrimination between $f(k, s)$ and $f^{\prime}(k, s)$ cannot be made. We arrive at the same conclusion in the course of our analysis of simulated data.

\section{Analysis of simulated data}

In this section, we show how we apply corrections to published experimental results on the parameters of narrow resonances based on fits to our simulated data. We deliberately consider only experiments listed in the 1986 Review of Particle Properties [1]. In changing values of the resonance parameters we strictly use information contained in the original experimental [14-18] and theoretical $[6,19,20]$ papers. References for the $\mathrm{J} / \psi, \psi^{\prime}, \Upsilon(1 S), \Upsilon(2 S)$ and $\Upsilon(3 S)$ are given in [14], [15], [16], [17] and [18] respectively. In ref. [19,20] the virtual terms are treated correctly but because the nature of the $\mathrm{J} / \psi$ was not established at the time, the vacuum polarization contributions are not explicitly included.

In order to correct measurements based on data we cannot access and which have been corrected with eq. (5), we use the simulated data as described in the previous section. We generate cross section data as a function of energy according to eq. (1) and eq. (3), with experimental parameters such as energy resolution equal to those of the published papers. We then fit the data to the convoluted cross sections obtained using the functions $f(k, s)$ and $f^{\prime}(k, s)$. The four free parameters of the fit are $M$ and $\Gamma$, the mass and the total width of the resonance, the beam energy spread, $\sigma_{\mathrm{E}}$, and the non-resonant cross section, $\sigma_{\text {nonres }}$. Thus, $B_{\mathrm{e}}$, the branching ratio into electrons, is fixed at the world average value [1].

In correcting published values of the resonance parameters, we take account of the fact that experiments differ from one another in several significant ways:

1. Note that $\mathrm{e}^{+} \mathrm{e}^{-}$storage rings differ in their energy resolution. In the $3 \mathrm{GeV}$ range, SPEAR, ADONE, and DORIS have roughly the same resolution, $\sigma_{\mathrm{E}} \approx 1$ $\mathrm{MeV}$. Around $10 \mathrm{GeV}, \mathrm{CESR}$ and VEPP IV have an energy resolution of $\sigma_{\mathrm{E}} \approx 4$ $\mathrm{MeV}$, while DORIS has $\sigma_{\mathrm{E}} \approx 8 \mathrm{MeV}$. In a comparison between our procedure and other radiative correction procedures, a change in $\sigma_{\mathrm{E}}$ from $4 \mathrm{MeV}$ to $8 \mathrm{MeV}$ increases the correction to $\Gamma_{\mathrm{e}}^{0}$ by $0.5 \%(C=1)$. We take this effect into account. Fig. 3 shows the dependence of the $\Gamma_{\mathrm{e}}^{0}$ correction on the ratio $C$, for $\sigma_{\mathrm{E}}=4 \mathrm{MeV}$ and $\sigma_{\mathrm{E}}=8 \mathrm{MeV}$.

2. In different experiments, the percentages $p$ of the total luminosity collected on the resonance peak, as compared to below or above the peak, can vary substantially. To understand the effect of this difference in the distribution of the data on the measurement of the mass and width, we have generated, for a given $\sigma_{E}$, two data sets, one with a fraction $p \approx 0.3$ and the other with $p \approx 0.7$ of the luminosity assigned to the peak of the resonance. For this purpose the peak region is defined as $M \pm 2 \sigma_{\mathrm{E}}$. For the experiments considered, the accumulated data are distributed 


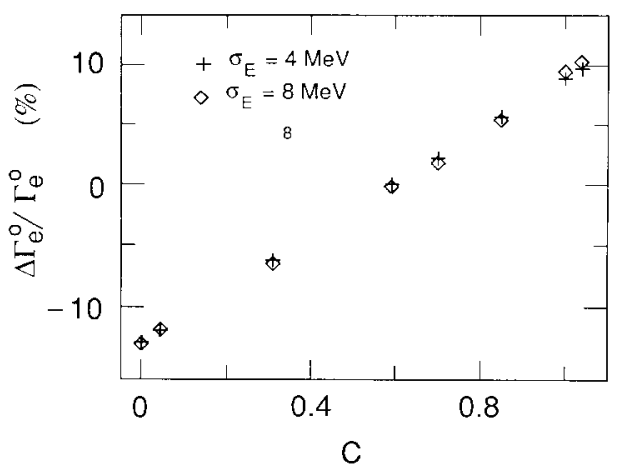

Fig. 3. Dependence of the $\Gamma_{\mathrm{e}}^{0}$ correction on the ratio $C$ at the $\Upsilon(9640)$, for $\sigma_{\mathrm{E}}=8 \mathrm{MeV}$ (diamonds) and $\sigma_{\mathrm{E}}=4 \mathrm{MeV}$ (crosses).

within these limits. The correction to the partial width $\Gamma_{\mathrm{e}}^{0}$, for $C=1$, increases by $1 \%$ when we vary the fraction $p$ from 0.3 to 0.7 . For every experiment under study, we evaluate the fraction $p$ and then obtain the specific correction by interpolation between the results for $p=0.3$ and $p=0.7$. The error associated with this procedure is estimated to be less than $0.5 \%$. Fig. 4 shows the dependence of the $\Gamma_{\mathrm{e}}^{0}$ correction on the ratio $C$, for samples with $p=0.3$ and $p=0.7$.

3. Most of the measurements on the $\psi$ and $\Upsilon$ resonances have been radiatively corrected based on the prescription by Jackson and Scharre [6]. For these, we typically derive changes in $\Gamma_{\mathrm{e}}^{0}$ of $2 \%$ at the $T(9460)$ by fits to simulated data. One experiment, unfortunately, added the full and correct vacuum polarization to $f^{\prime}(k, s)$, and this resulted in a large correction to $\Gamma_{\mathrm{e}}^{0}$ of $\approx 9 \%$. Two other experiments derived resonance parameters using algorithms $[19,20]$ which are identical to ours, except for the vacuum polarization and the $\delta_{2}$ terms.

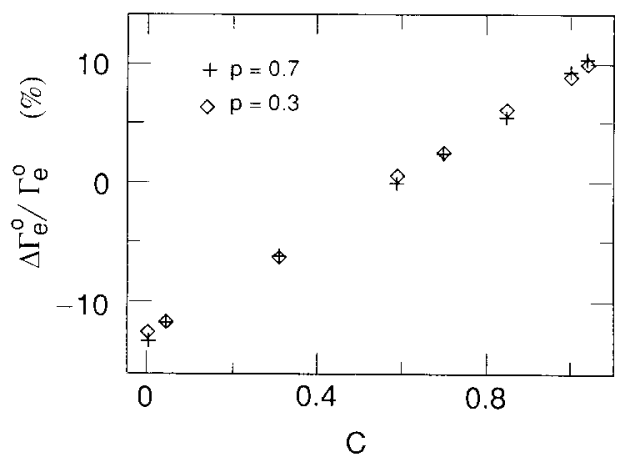

Fig. 4. The correction $\Delta \Gamma_{\mathrm{e}}^{0} / \Gamma_{\mathrm{e}}^{0}$ as a function of the ratio $C$ at the $\Upsilon(9460)$ for data samples with $p=0.3$ (diamonds) and $p=0.7$ (crosses) for $\sigma_{\mathrm{E}}=4 \mathrm{MeV}$. 
In summary, the fact that the changes to the resonance parameters vary from experiment to experiment is almost completely due to the differences in the radiative corrections that the various experimenters applied. The energy resolution and the statistical spread of the data have only small effect on the variation between experiments.

If the fitting formula factorizes the virtual terms as in $f(k, s)$, then missing vacuum polarization terms only enter as a correction to the normalization. This means that the fit predicts the mass correctly, but it underestimates the area under the resonance by a fraction approximately equal to $\delta_{\mathrm{h}}+\delta_{\mu}+\delta_{\tau}+\delta_{2}$.

If the fitting formula separates real and virtual terms as in $f^{\prime}(k, s)$, the problem is more complicated. The dependence of the correction on the ratio $C$ is best found by fits to simulated data. When full vacuum polarization is used, i.e. $C \approx 1.03-1.04$, we find a systematic increase in the mass, the non-resonant cross section, and the beam energy spread, and a decrease in the leptonic width. This is consistent with fig. 1, and was also observed in simulated data at the $\mathrm{Z}^{0}$ resonance [2], where $C=1$. When the virtual terms in $f^{\prime}(k, s)$ are set to zero, which is equivalent to setting $\delta_{\text {tot }}=0$ in eq. (10), then $C=0$ and $f(k, s)$ and $f^{\prime}(k, s)$ are identical in shape (the hard term is not significant), but their normalizations differ by a factor $1+\delta_{1}+\delta_{2}+\delta_{\mathrm{vp}}+K$. This will produce no change in the mass, but will increase the leptonic width. Intermediate values of $C$ will generate corrections to the leptonic width and mass as displayed in figs. 5 and 6 . The shift in the mass $\Delta M$ is normalized to the energy resolution $\sigma_{E}$, because we find empirically that for a fixed ratio $C$ the mass shift is proportional to $\sigma_{\mathrm{E}}$. This behavior is attributed to the fact that the equivalent radiator thickness $\beta$ is the same at the $\psi$ and $\gamma$ to within $10 \%$, and because $f(k, s)$ has a very similar shape for the two resonance families. Notice also that above $C=0.8, \Delta M / \sigma_{\mathrm{E}}$ remains constant. These curves can be used to correct experimental results which are not listed here.

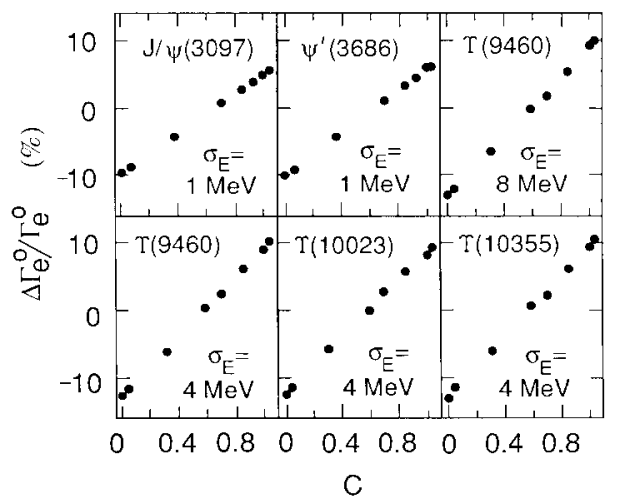

Fig. 5. Corrections to $I_{\mathrm{c}}^{0}$ versus $C$ for the five narrow resonances of the $\psi$ and $\Upsilon$ families. The corrections to the $\Upsilon(10023)$, are roughly equal for $\sigma_{\mathrm{E}}=8 \mathrm{MeV}$ and for $\sigma_{\mathrm{E}}=4 \mathrm{MeV}$. 


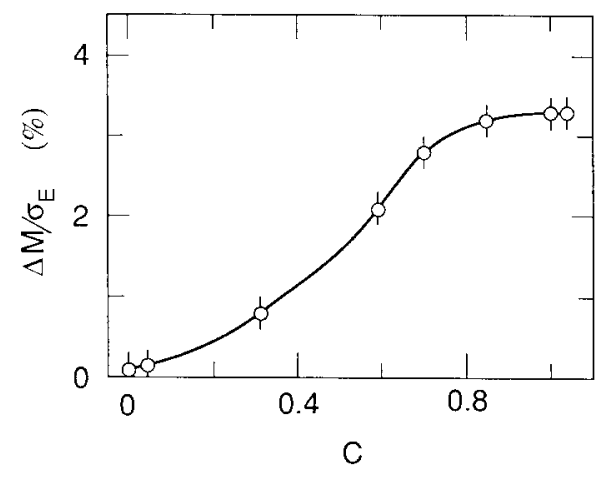

Fig. 6. Corrections to the mass $M$ as a function of the ratio $C . \Delta M$ is given in units of the machine resolution $\sigma_{\mathrm{E}}$.

TABLE 1

Summary of the corrections to the parameters of $\psi$ and $\Upsilon$ resonances, listed by experiment

\begin{tabular}{|c|c|c|c|c|}
\hline Quantity & Ref. & $\begin{array}{c}\text { New value } \\
\Gamma_{\mathrm{c}}^{0}\end{array}$ & $\begin{array}{c}\text { New value } \\
\Gamma_{\mathrm{e}}^{\exp }\end{array}$ & Old value \\
\hline$\Gamma_{\mathrm{e}}, \mathrm{J} / \psi(3097)$ & Boyarski [14] & $4.6 \mathrm{keV}$ & $4.8 \mathrm{keV}$ & $4.8 \mathrm{keV}$ \\
\hline$\Gamma_{\mathrm{e}}, \mathrm{J} / \psi(3097)$ & Baldini [14] & $4.5 \mathrm{keV}$ & $4.7 \mathrm{keV}$ & $4.6 \mathrm{keV}$ \\
\hline$\Gamma_{c}, \mathrm{~J} / \psi(3097)$ & Esposito [14] & $4.5 \mathrm{keV}$ & $4.7 \mathrm{keV}$ & $4.6 \mathrm{keV}$ \\
\hline$\Gamma_{c}, \mathrm{~J} / \psi(3097)$ & Brandelik [14] & $4.5 \mathrm{keV}$ & $4.6 \mathrm{keV}$ & $4.4 \mathrm{keV}$ \\
\hline$\Gamma_{c}, \psi(3685)$ & Lüth [15] & $2.0 \mathrm{keV}$ & $2.1 \mathrm{keV}$ & $2.1 \mathrm{keV}$ \\
\hline$\Gamma_{\mathrm{e}}, \psi(3685)$ & Brandelik [15] & $2.1 \mathrm{keV}$ & $2.2 \mathrm{keV}$ & $2.0 \mathrm{keV}$ \\
\hline$\Gamma_{\mathrm{e}}, \Upsilon(9460)$ & Berger [16] & $1.36 \mathrm{keV}$ & $1.46 \mathrm{keV}$ & $1.33 \mathrm{keV}$ \\
\hline$\Gamma_{\mathrm{c}}, \Gamma_{(9460)}$ & Bock $[16]$ & $1.10 \mathrm{keV}$ & $1.18 \mathrm{keV}$ & $1.08 \mathrm{kcV}$ \\
\hline$I_{\mathrm{e}}, \Upsilon(9460)$ & Albrecht [16] & $1.25 \mathrm{keV}$ & $1.34 \mathrm{keV}$ & $1.23 \mathrm{keV}$ \\
\hline$\Gamma_{\mathrm{e}}, \Upsilon(9460)$ & Niczyporuk [16] & $1.15 \mathrm{keV}$ & $1.24 \mathrm{keV}$ & $1.13 \mathrm{kcV}$ \\
\hline$\Gamma_{\mathrm{e}}, \Upsilon(9460)$ & Tuts [16] & $1.18 \mathrm{keV}$ & $1.27 \mathrm{keV}$ & $1.15 \mathrm{keV}$ \\
\hline$\Gamma_{\mathrm{e}}, \Upsilon(9460)$ & Giles [16] & $1.42 \mathrm{keV}$ & $1.53 \mathrm{keV}$ & $1.30 \mathrm{keV}$ \\
\hline$\Gamma_{\mathrm{e}}, \Upsilon(10023)$ & Bock [17] & $0.40 \mathrm{keV}$ & $0.43 \mathrm{keV}$ & $0.39 \mathrm{keV}$ \\
\hline$\Gamma_{\mathrm{c}}, \Upsilon(10023)$ & Niczyporuk [17] & $0.58 \mathrm{keV}$ & $0.62 \mathrm{keV}$ & $0.56 \mathrm{keV}$ \\
\hline$\Gamma_{c}, \Upsilon(10023)$ & Albrecht [17] & $0.60 \mathrm{keV}$ & $0.65 \mathrm{keV}$ & $0.58 \mathrm{keV}$ \\
\hline$\Gamma_{\mathrm{c}}, \Upsilon(10023)$ & Tuts [17] & $0.58 \mathrm{keV}$ & $0.62 \mathrm{keV}$ & $0.56 \mathrm{keV}$ \\
\hline$I_{\mathrm{e}}, T(10023)$ & Giles [17] & $0.57 \mathrm{keV}$ & $0.61 \mathrm{keV}$ & $0.52 \mathrm{keV}$ \\
\hline$\Gamma_{\mathrm{e}}, \Upsilon(10355)$ & Tuts [18] & $0.40 \mathrm{keV}$ & $0.32 \mathrm{keV}$ & $0.39 \mathrm{keV}$ \\
\hline$\Gamma_{\mathrm{e}}, \Gamma(10355)$ & Giles [18] & $0.46 \mathrm{keV}$ & $0.49 \mathrm{keV}$ & $0.42 \mathrm{keV}$ \\
\hline$M, \Upsilon(9460)$ & Artamonov [16] & $9460.5 \mathrm{MeV}$ & - & $9460.6 \mathrm{MeV}$ \\
\hline$M, \Upsilon(9460)$ & Mac Kay [16] & $9459.87 \mathrm{MeV}$ & - & $9459.97 \mathrm{MeV}$ \\
\hline
\end{tabular}


TABLE 2

New world averages for those resonance parameters which change by more than $50 \%$ of a standard deviation. Also given are the percentage change in the experimental quantities, and the statistical significance of the change in units of overall experimental error

\begin{tabular}{|c|c|c|c|c|}
\hline Quantity & $\begin{array}{c}\text { New world } \\
\text { average [keV] }\end{array}$ & $\begin{array}{c}\text { New world } \\
\text { average, } \Gamma_{\mathrm{c}}=\Gamma_{\mu}\end{array}$ & $\begin{array}{l}\text { Fractional } \\
\text { change }\end{array}$ & $\begin{array}{c}\text { Statistical } \\
\text { change }\end{array}$ \\
\hline$\Gamma_{\mathrm{e}}^{0}, \mathrm{~J} / \psi(3097)$ & $4.57 \pm 0.51 \mathrm{keV}$ & $4.53 \pm 0.35 \mathrm{keV}$ & $-4.0 \%$ & $0.5 \sigma$ \\
\hline$\Gamma_{\mathrm{e}}^{0}, \psi(3685)$ & - & $2.05 \pm 0.21 \mathrm{keV}$ & 0 & 0 \\
\hline$\Gamma_{\mathrm{c}}^{0}, \Upsilon(9460)$ & - & $1.279 \pm 0.050 \mathrm{keV}$ & $4.5 \%$ & $1.1 \sigma$ \\
\hline$\Gamma_{\mathrm{c}}^{0}, \Upsilon(10023)$ & - & $0.569 \pm 0.033 \mathrm{keV}$ & $6.0 \%$ & $1.0 \sigma$ \\
\hline$\Gamma_{\mathrm{e}}^{0}, \Gamma(10355)$ & - & $0.423 \pm 0.031 \mathrm{keV}$ & $5.2 \%$ & $0.7 \sigma$ \\
\hline$\Gamma_{\mathrm{c}}^{\exp }, \mathrm{J} / \psi(3097)$ & $4.77 \pm 0.51 \mathrm{keV}$ & $4.72 \pm 0.35 \mathrm{keV}$ & $+0.4 \%$ & $0.1 \sigma$ \\
\hline$\Gamma_{\mathrm{e}}^{\exp }, \psi(3685)$ & - & $2.14 \pm 0.21 \mathrm{keV}$ & $4.4 \%$ & $0.4 \sigma$ \\
\hline$\Gamma_{\mathrm{e}}^{\mathrm{exp}}, \Upsilon(9460)$ & - & $1.376 \pm 0.050 \mathrm{keV}$ & $12.4 \%$ & $3.0 \sigma$ \\
\hline$\Gamma_{\mathrm{e}}^{\mathrm{exp}}, \Upsilon(10023)$ & - & $0.612 \pm 0.033 \mathrm{keV}$ & $14.0 \%$ & $2.3 \sigma$ \\
\hline$\Gamma_{\mathrm{e}}^{\mathrm{exp}}, \Upsilon(10355)$ & - & $0.455 \pm 0.031 \mathrm{keV}$ & $13.2 \%$ & $1.7 \sigma$ \\
\hline$\Gamma, \Upsilon(9460)$ & - & $48.5 \pm 3.2 \mathrm{keV}$ & $12.6 \%$ & $1.7 \sigma$ \\
\hline$\Gamma, \Upsilon(10023)$ & - & $34.2 \pm 7.3 \mathrm{keV}$ & $14.0 \%$ & $0.6 \sigma$ \\
\hline$M, \Upsilon(9460)$ & $9459.93 \pm 0.19 \mathrm{MeV}$ & - & $0.001 \%$ & $0.5 \sigma$ \\
\hline
\end{tabular}

Table 1 lists the values of the masses and widths of the $\psi$ and $\Upsilon$ resonances from experiments referenced in the 1986 Review of Particle Properties [1]. Both previously measured and refitted values are given. We would like to point out that our method is one of simulation; it shows fluctuations of typically $2-3 \%$ in the fitted parameters when cross sections are assigned errors that are comparable to those in published experiments. Likewise, fits performed on the real data from a given experiment can deviate from our values at the few percent level. The overall error of our method, based on much smaller point to point errors, is conservatively estimated to be $1 \%$. It is to be added in quadrature to the theoretical error discussed in sect. 1 .

Using the corrections to $\Gamma_{\mathrm{e}}^{0}$, we have derived the corrections to $\Gamma$ taking into account the error on the branching ratio. We decouple the measurement of $\Gamma_{\mathrm{c}}^{0}$ and $\Gamma$ by consistently using the world average branching ratio, ${ }^{\star}$ and not the particular value as measured by a given experiment. It can be easily shown that $\Gamma_{\mathrm{e}}^{0}$ and $B_{\mathrm{c}}$ can have both independent and correlated sources of systematics within the same experiment, and our method tends to eliminate such possible correlation. New and more precise measurements of the leptonic branching ratios could induce significant changes in the values of the total widths.

Table 2 contains the summary of our results, presented in the form of new world averages for the resonance parameters that change significantly with our new

* We use branching ratios from ref. [1]. Refs. [22] and [23] also use a single average branching ratio to obtain $\Gamma$, but more recent measurements are included there. 
analysis. Quantities wnch do not change the world average by at least $50 \%$ of a standard deviation are not listed, although we have included the leptonic widths for all five narrow resonances. We have only included in the analysis resonances which are below the threshold for open flavor production. The corrections to resonance parameters above this threshold resemble the corrections discussed for the $Z^{0}$ [2], and are small. This is true even for the $\Upsilon(4 S)$, which has a shape that has been measured with far higher precision than any other open flavor resonance. In this case, the corrections to all three resonance parameters are less than $50 \%$ of the overall experimental error.

\section{Conclusions and discussion}

In conclusion, we have applied an improved prescription for QED radiative corrections to narrow resonance production in $\mathrm{e}^{+} \mathrm{e}^{-}$annihilation. The estimated uncertainty of $1 \%$ in the $3-10 \mathrm{GeV}$ region of center of mass energy is dominated by the understanding of the hadron part of the photon vacuum polarization [21] ${ }^{\star}$. The methodology used here for the re-fitting of $\psi$ and $\Upsilon$ states takes into account differences in the data of the various experiments. The observed shifts of the mass, total width, and electron partial width of these resonances are small, but when we combine the new values for all experiments and form new world averages, the changes are significant. The values of several quantities change as a result of our reevaluation of the radiative corrections by up to three standard deviations. The implications of the reanalysis of $\psi$ and $r$ states for quarkonium potential models have been discussed elsewhere $[22,23]^{\star \star}$.

Recently two other papers $[22,23]$ have dealt with the subject of radiative corrections to narrow resonances. Both use a formulation that is consistent with eq. (3). However, Buchmüller and Cooper [23] rescale the results for the $\Upsilon$ states using only the peaks of the resonances, thereby obtaining changes to world averages which are slightly larger than ours. The correction method of Königsmann [22] gives results for the $T$ resonances which are nearly identical to those of ref. [23]. However, his results for the $\psi$ states differ substantially from ours, and we believe that this is because our method of simulating cross section data correctly accounts for the various nontrivial effects arising from a resonance fit. As discussed in sect. 2, the $\mathrm{J} / \psi$ and $\psi^{\prime}$ resonance data are more sensitive to these effects than are the $\Upsilon$ data.

\footnotetext{
* Since $\delta_{\mathrm{h}}$ is calculated from a dispersion relation integral which includes low energy $\mathrm{c}^{+} \mathrm{e}^{-} \rightarrow$ hadrons data, then a better understanding of the $\psi$ and $r$ families could, in principle, reduce the crror on $\delta_{\mathrm{h}}$ at $\sqrt{s} \leqslant 100 \mathrm{GeV}$, which may ultimately limit tests of the Standard Model at the $Z^{0}$ resonance. However, our analysis has a negligible impact on the overall error for $\delta_{\mathrm{h}}$ of 3-6\%, as determined in the recent calculations of ref. [21].

** We do not support the statement that the treatment by Kuraev and Fadin does not include vacuum polarization [see eq. (6), ref. [4]]. However, this is not relevant for the analysis of this paper.
} 
We would like to thank L. Trentadue for useful discussions, and S. Cooper for helpful suggestions.

\section{Appendix A}

We use in our analysis the form of the distribution function $f(k, s)$ from eq. (3) which has been convoluted [see eq. (1)] analytically with a Breit-Wigner according to Cahn [11]. We add, however, the $\delta_{2}$ and $\delta_{\mathrm{vp}}$ terms given below. We then convolute this result, $g(s)$, with a gaussian energy resolution function.

$$
\begin{aligned}
g(s)= & \sigma_{1}\left(1+\delta_{1}+\delta_{2}\right) \frac{\Gamma^{2}}{\Gamma^{2}+M^{2}}\left[\frac{s}{M^{2}} a^{\beta-2} \Phi(\cos \theta, \beta)-a^{\beta-1} \frac{\beta}{1+\beta} \Phi(\cos \theta, 1+\beta)\right] \\
& -\sigma_{1} \beta \frac{\Gamma}{\sqrt{s}}\left[\tan ^{-1} \frac{2 M}{\Gamma}-\tan ^{-1} \frac{2(M-\sqrt{s})}{\Gamma}\right]
\end{aligned}
$$

where

$$
\sigma_{1}=\sigma_{\text {peak }}\left(1+\delta_{\mathrm{vp}}\right)(1+K)
$$

Ref. (4) yields the result:

$$
\begin{aligned}
& \delta_{1}=\frac{3}{4} \beta \\
& \delta_{2}=-\frac{\beta^{2}}{24}\left(\frac{1}{3} \log \frac{s}{m_{\mathrm{e}}^{2}}+2 \pi^{2}-\frac{37}{4}\right) .
\end{aligned}
$$

The $K$-factor is defined as ( $\alpha$ is the fine structure constant)

$$
K=\frac{\alpha}{\pi}\left(\frac{\pi^{2}}{3}-\frac{1}{2}\right)
$$

The vacuum polarization term is given by the sum of the loops over leptons and hadrons,

$$
\delta_{\mathrm{vp}}=\delta_{\ell}+\delta_{\mathrm{h}}, \text { with } \delta_{\ell}=\delta_{\mathrm{e}}+\delta_{\mu}+\delta_{\tau}
$$

The vacuum polarization contribution of charged leptons of mass $m_{i}$ is

$$
\delta_{\ell}=-\sum_{i} \frac{2 \alpha}{\pi}\left(\frac{5}{9}+\frac{1}{3} \log \frac{m_{i}^{2}}{s}\right)
$$


The quantities $a, \cos \theta$, and $\Phi(\cos \theta, \beta)$ are defined as follows:

$$
\begin{aligned}
a^{2} & =\frac{M^{2}\left(s / M^{2}-1\right)^{2}+\Gamma^{2}\left(s / M^{2}\right)^{2}}{\Gamma^{2}+M^{2}} ; \\
\cos \theta & =-\frac{M^{2}\left(s / M^{2}-1\right)+\Gamma^{2}\left(s / M^{2}\right)}{a\left(\Gamma^{2}+M^{2}\right)} ; \\
\Phi(\cos \theta, \beta) & =\frac{\pi \beta \sin ((1-\beta) \theta)}{\sin \theta \beta \sin \theta} .
\end{aligned}
$$

\section{References}

[1] Particle Data Group, M. Aquilar-Beniter et al., Review of particle properties, Phys. Lett. B170 (1986) 1

[2] J. Alexander et al., Phys. Rev. D37 (1988) 56

[3] T. Kinoshita, J. Math. Phys. 3 (1962) 650;

T.D. Lee and M. Nauenberg, Phys. Rev. B133 (1964) 1549

[4] E.A. Kuraev and V.S. Fadin, Sov. J. Nucl. Phys. 41(3) (1985) 466

[5] F.A. Berends and G.J. Komen, Phys. Lett. B63 (1976) 432

[6] J.D. Jackson and D.L. Scharre, Nucl. Instr. and Meth. 128 (1975) 13

F.A. Berends and G.J. Komen, Nucl. Phys. B115 (1976) 114

[7] V.N. Gribov and L.N. Lipatov, Sov. J. Nucl. Phys. 15 (1972) 438

[8] E. Etim et al., Nuovo Cimento 51 (1967) 276

[9] F.A. Berends, G.J.H. Burgers and W.L. Van Neerven, Phys. Lett. B185 (1987) 395

[10] F. Bloch and A. Nordsieck, Phys. Rev. 52 (1937) 54

[11] R.N. Cahn, Phys. Rev. D36 (1987) 2666

[12] V.N. Baier et al., Phys. Rep. 78 (1981) 294;

Ya.I. Azimov et al., JETP Lett. 21 (1975) 378;

P. Tsai, SLAC-PUB-3129, unpublished

[13] A.M. Boyarski et al., Phys. Rev. Lett. 34 (1975) 1357:

V. Lüth et al., Phys. Rev. Lett. 35 (1975) 1124

[14] A.M. Boyarski et al., Phys. Rev. Lett. 34 (1975) 1357;

R. Baldini-Celio et al., Phys. Lett. B58 (1975) 471;

B. Esposito et al., Nuovo Cimento Lett. 14 (1975) 73;

R. Brandelik et al., Z. Phys. C1 (1979) 233

[15] V. Lüth et al., Phys. Rev. Lett. 35 (1975) 1124;

R. Brandelik et al., Z. Phys. C1 (1979) 233

[16] A.S. Artamonov et al., Phys. Lett. B137 (1984) 272;

W.W. MacKay et al.. Phys. Rev. D29 (1984) 2483;

C. Berger et al., Z. Phys. Cl (1979) 343;

B. Bock et al., Z. Phys. C6 (1980) 1125;

H. Albrecht et al., Phys. Lett. B116 (1982) 383;

B. Niczyporuk et al, Z. Phys. C15 (1982) 299;

P.M. Tuts et al, 1983 Int. Symposium on Lepton and Photon Interactions (Cornell, NY) p. 248;

R.T. Giles et al., Phys. D29 (1984) 1285 
[17] B. Bock et al., Z. Phys. C6 (1980) 1125;

H. Albrecht et al., Phys. Lett. B116 (1982) 383;

B. Niczyporuk et al., Phys. Lett. B99 (1981) 169;

P.M. Tuts et al., 1983 Int. Symposium on Lepton and Photon Interactions (Cornell, NY) p. 248;

R.T. Giles et al., Phys. Rev. D29 (1984) 1285

[18] P.M. Tuts et al., 1983 Int. Symposium on Lepton and Photon Interactions (Cornell, NY) p. 248;

R.T. Giles et al., Phys. Rev. D29 (1984) 1285

[19] M. Greco et al., Phys. Lett. B56 (1975) 367

[20] Y.S. Tsai, SLAC-PUB-1515 (1974)

[21] B.W. Lynn, G. Penso and C. Verzegnassi, Phys. Rev. D35 (1987) 42;

F. Jegerlehner, Z. Phys. C32 (1986) 195

[22] K. Königsmann, DESY 87-046 (1987)

[23] W. Buchmüller and S. Cooper, MIT-LNS-159 (1987) 